\title{
Análisis de la variabilidad alélica de BmVDAC y Subolesina, dos candidatos vacunales contra Rhipicephalus microplus en aislados de México Analysis of the allelic variability of BmVDAC and Subolesin, two vaccine candidates against Rhipicephalus microplus in isolates from Mexico
}

\author{
María Martina Esperanza Pérez Soria ${ }^{1}$, Diego Josimar Hernández Silva ${ }^{1}$ y Juan Mosqueda ${ }^{1}$
}

Palabras clave: garrapatas; Rhipicephalus microplus; antígenos; variabilidad antigénica; vacuna Keywords: ticks; Rhipicephalus microplus; antigen; antigenic variability; vaccine

Recibido en: 29-04-2019 / Aceptado en: 05-07-2019

\section{Resumen}

Introducción: Las garrapatas Rhipicephalus microplus son ectoparásitos hematófagos que impactan económicamente la ganadería a nivel mundial. Los acaricidas han sido una estrategia de control efectiva pero que generan la resistencia a estos químicos y la contaminación del medio ambiente. Una alternativa para contrarrestar los daños adversos de los químicos es la utilización de vacunas, las cuales presentan eficacia variable, debido posiblemente a la diferencia en la secuencia de aminoácidos entre los antígenos de las garrapatas de diferentes zonas geográficas. Por lo que el objetivo del presente trabajo fue analizar la variabilidad de dos antígenos vacunales: BmVDAC (Voltage Dependent Anion Channel) y Subolesina de $R$. microplus en aislados provenientes de diferentes estados de México.

Método: Dos proteínas se seleccionaron con base a su eficacia como antígenos protectores: BmVDAC y Subolesina. Se colectaron garrapata $R$. microplus de distintos estados de México. Se realizaron extracciones ADN y ARN para la amplificación de bmvdac y subolesina respectivamente. Se obtuvieron las secuencias predichas de aminoácidos. Se hizo un análisis BLAST para confirmar la identidad entre las secuencias y alinearlas con el programa Clustal Omega para determinar el grado de similitud. Finalmente, se realizó un análisis con el programa MEGAX64 para determinar la relación filogenética de cada aislado.

Resultados: Para BmVDAC, se observó un porcentaje promedio de identidad y similitud del 99.79\% entre las diferentes secuencias de aminoácidos. En aislados de Chiapas, Nayarit, Media Joya, Querétaro, Tamaulipas y Guerrero se observó un porcentaje de identidad y similitud del 99.87\%. Jalisco, Tabasco y Sinaloa presentaron el $99.56 \%$ en identidad y similitud. En promedio se observa un porcentaje de variabilidad de $0.21 \%$.

\footnotetext{
${ }^{1}$ Universidad Autónoma de Querétaro, Facultad de Ciencias Naturales. Querétaro, Querétaro. E-mail: joel.mosqueda@uaq.mx

(C) Universidad De La Salle Bajío (México)
} 
En el alineamiento de Subolesina con aislados de Yucatán, Nayarit, Querétaro, Chiapas, Sinaloa, Veracruz y Tamaulipas, y la cepa Munoz, se observó un porcentaje promedio de identidad del 99.87\% y similitud del 100\% entre las secuencias de aminoácidos. De las ocho secuencias, siete presentaron procentaje de identidad del $99.91 \%$, mientras que para Nayarit el porcentaje fue de 99.39\%. La variabilidad promedio fue del $0.13 \%$. Los análisis filogenéticos no mostraron diferencias significativas entre las secuencias utilizando el método UPGMA. El porcentaje promedio obtenido del método Bootstrap de 10000 réplicas para los árboles filogenéticos de bmvdac y subolesina fue de más del $50 \%$.

Discusión o Conclusión: Este reporte representa la primera investigación respecto al grado de conservación entre aislados de $R$. microplus de diferentes estados en México para BmVDAC y Subolesina, dos candidatos vacunales contra esta garrapata. Considerando la variabilidad presentada entre las secuencias de los diferentes aislados de $R$. microplus, se puede concluir que BmVDAC y Subolesina son antígenos que se encuentran conservados, por lo que pueden considerarse como candidatos vacunales. Esta información es relevante para la selección de antígenos empleados en vacunas anti-garrapatas $R$. microplus en México.

\begin{abstract}
Introduction: Rhipicephalus microplus ticks are hematophagous ectoparasites that economically impact lifestock industry worldwide. Acaricides have been an effective control strategy, but generate resistance to chemical compounds and environmental pollution. An alternative to counteract the chemical adverse effects is the vaccination usage, which is effective, environmentally friendly and unexpensive. However, still anti-tick vaccines present variable efficacy due to possible differences in aminoacid sequences within tick antigens from geografically distinct isolates. The aim of this work was to analyze the variability of two vaccine antigens: BmVDAC (Voltage Dependent Anion Channel) and Subolesin from R. microplus isolates from different states of Mexico.

Methods: Two proteins were selected based on its efficacy as protective antigens: BmVDAC and Subolesin. R. microplus ticks was collected from different states of Mexico. Isolation of both DNA and RNA was performed to amplify bmvdac and subolesin respectively. Predicted amino acid sequences were obtained. A BLAST analysis was carried out to confirm the identity within
\end{abstract}


sequences and determine similarity by an alignment using Clustal Omega. Finally, to determine the phylogenetic relationship of each isolate an analysis by MEGAX64 software was performed. Results: For BmVDAC, an average percentage of $99.79 \%$ identity and similarity was observed within the different aminoacid sequences. In the isolates from Chiapas, Nayarit, Media Joya, Queretaro, Tamaulipas and Guerrero a similarity and identity percentage of $99.87 \%$ was observed. Jalisco, Tabasco and Sinaloa a similarity and identity percentage of $99.56 \%$ was presented. On average, a variability percentage of $0.21 \%$ was observed.

The Subolesin alignment with isolates from Yucatan, Nayarit, Queretaro, Chiapas, Sinaloa, Veracruz, Tamaulipas, and the reference Munoz strain, an identity average percentage of $99.87 \%$ and similarity percentage of $100 \%$ was observed in the aminoacid sequences. Seven from eight sequences presented identity percentage of $99.91 \%$ whereas for Nayarit the percentage was 99.39\%. The average variability was $0.13 \%$. Phylogenetic analysis did not show significant differences between sequences using the UPGMA method. Average percentage obtained by 10000 replicas by Bootstrap method for bmvdac and subolesin was up to $50 \%$.

Discussion and conclusion. This report represents the first research on the characterization of the variability for BmVDAC and Subolesin, two vaccine antigens against $R$. microplus isolates from different states of Mexico. Considering the low variability presented by the different $R$. microplus isolates sequences, we can conclude that BmVDAC and Subolesin are antigens that are conserved and can be considered as vaccine candidates. This information is relevant for antigen selection and vaccine design against $R$. microplus in Mexico.

\section{Introducción}

Las garrapatas ocasionan pérdidas económicas en el ganado a nivel mundial, parasitan una amplia gama de huéspedes vertebrados y transmiten una gran variedad de agentes patógenos, más que cualquier otro grupo de artrópodos (Prudencio et al., 2010).

La garrapata Rhipicephalus microplus se alimenta de la sangre del ganado bovino, su hospedero definitivo, y es considerada como uno de los parásitos más dañinos en el ganado de zonas tropicales y subtropicales (Antunes et al., 2014).

La infestación por garrapata $R$. microplus afecta negativamente la productividad del ganado, transmite enfermedades tales como la babesiosis y la anaplasmosis (Jonsson, 2006). Las 
pérdidas económicas anuales en la industria ganadera son estimadas entre veintidós a treinta billones de dólares (Tabor et al., 2016), las cuales son resultado de la actividad directa en la alimentación de las garrapatas en su hospedero y del costo que ocasiona controlar tanto a las garrapatas como a las enfermedades producidas por los patógenos que ellas transmiten (Manjunathachar et al., 2014).

El método tradicional para el control de garrapatas está basado en el uso de químicos acaricidas a los cuales las garrapatas han desarrollado resistencia (Graf et al., 2004). Esto dificulta la erradicación y el control de las garrapatas, por lo que el uso de productos químicos no representa la mejor opción (Sonenshine y Roe, 2013). Tomando en cuenta la creciente preocupación por la seguridad del medio ambiente, la salud humana y la salud animal, se han vuelto necesarias las medidas de control alternativas (Abbas et al., 2014). Actualmente una estrategia para el control de la garrapata es el desarrollo de la inmunidad en el hospedero al administrar antígenos de la garrapata por medio de vacunas (Walker, 2009). La vacunación es considerada como una alternativa rentable, amigable con el medio ambiente, no genera contaminación residual en los productos de origen animal, es de fácil aplicación y es tecnología económica (Jonsson, 2006).

En los años noventa aparecieron comercialmente las vacunas contra la garrapata del ganado (Kaewmongkol et al., 2015). Estas vacunas están basadas en la forma recombinante del antígeno oculto Bm86 del intestino de $R$. microplus (García-García et al., 1998). Sin embargo, la eficacia de esta vacuna varía de acuerdo a los aislados existentes en las diferentes zonas geográficas (de la Fuente et al., 2007), lo cual puede ser debido a variaciones alélicas en el gen bm86 (De La Fuente y Kocan, 2006) (Sossai et al., 2005). Una diferencia del 3.4\% en la secuencia de Bm86 entre las diferentes cepas puede ser suficiente para causar una respuesta inmune ineficiente (García-García et al., 1999). Se sugiere que existe una correlación inversa entre la eficacia de la vacuna y la variación del locus de bm86 (Freeman et al., 2010). Todo esto sugiere que un candidato vacunal debe ser conservado en su secuencia de aminoácidos entre distintas cepas o aislados para poder ser eficaz.

Existe un gran número de proteínas de garrapatas que han sido evaluadas como componentes para las vacunas, obteniendo diferentes porcentajes de protección, entre las que se encuentran BmVDAC y Subolesina (Taheri et al., 2014). La proteína BmVDAC está presente en las células del intestino de la garrapata, es una porina mitocondrial, interviene en regulación de la 
apoptosis en células infectadas, y tiene una sobre expresión importante durante la invasión de Babesia bigemina (Rodríguez-Hernández et al., 2015). La proteína Subolesina se localiza en diferentes órganos de la garrapata como los ovarios, las glándulas salivales y el intestino. Además, interviene en la digestión de la sangre, la reproducción y el desarrollo de la garrapata (de la Fuente et al., 2008). Un aspecto importante en la selección de antígenos que protejan contra las garrapatas, es la evaluación de la variabilidad alélica de los candidatos vacunales en las cepas de garrapatas de diferentes zonas geográficas; la identificación de secuencias peptídicas conservadas en estos candidatos vacunales permitirá obtener una vacuna con una protección elevada contra las diferentes cepas (de la Fuente y Kocan, 2003) (Manjunathachar et al., 2014). Derivado de lo anterior, el objetivo del presente trabajo fue analizar la variabilidad alélica en las secuencias de los antígenos vacunales BmVDAC y Subolesina de $R$. microplus en cepas provenientes de diferentes estados de México.

\section{Método}

\section{Selección de proteínas de $R$. microplus}

Se seleccionaron dos proteínas con base en su eficacia como antígenos candidatos vacunales, las cuales fueron: BmVDAC y Subolesina. Se obtuvieron las secuencias de referencia de la base de datos National Center of Biotechnology Information (NCBI), con los números de acceso GU994210.1y ABA62328.1, respectivamente.

\section{1) Colección de garrapatas $R$. microplus de México}

Los aislados seleccionados de R. microplus fueron colectados de los estados de Jalisco, Tabasco, Chiapas, Nayarit, Querétaro, Tamaulipas, Guerrero y Sinaloa, además de la cepa de referencia Media Joya. Estos se utilizaron para amplificar el gen bmvdac. Para el gen subolesina, se utilizaron los aislados provenientes de Yucatán, Nayarit, Chiapas, Tamaulipas, Querétaro, Sinaloa y Veracruz, además de la cepa Munoz utilizada como referencia. Por lo menos 30 hembras adultas de garrapatas $R$. microplus vivas fueron colectadas de distintos ranchos de cada estado. Fueron llevadas al laboratorio donde fueron lavadas y desinfectadas con benzal al 10\%, se secaron con toallas de papel e inmediatamente fueron congeladas a -20C hasta su uso. 


\section{Diseño de iniciadores}

Para el diseño de iniciadores para amplificar cada uno de los genes seleccionados se utilizaron los programas, Primer3web versión 4.1.0 (http://primer3.ut.ee/); el programa Primer Blast de la base de datos National Center of Biotechnology Information (NCBI) (www.ncbi.nlm.nih.gov/tools/primer-blast/) y finalmente, el programa GENtle (http://gentle.magnusmanske.de/).

\section{Amplificación de los genes en los diferentes aislados}

Los iniciadores utilizados para la amplificación de los genes en los diferentes aislados de $R$. microplus fueron, para el gen bmvdac: 5' ATGGCTCCTCCGTGCTACGC 3' (sentido) y 5' CTTGTGTCCTCCCTGGTTGAA 3' (antisentido), utilizando como referencia la secuencia de bmvdac reportada en GenBank (cepa Media Joya) número de acceso GU994210.1. Para la amplificación del gen subolesina, la secuencia fue obtenida del GenBank (cepa Munoz) con número de acceso ABA62328.1. Los iniciadores utilizados fueron: 5' AAAGCGAACACATGACTGGG3' (sentido) y 5' TCCCGTATCTTGCTCTCTCG 3' (anti sentido). Para amplificar el gen bmvdac, el ADN genómico fue extraído de los ocho diferentes aislados de $R$. microplus de México mediante el kit DNeasy Blood \& Tissue (Quiagen, Hiden, Alemania), de acuerdo a las indicaciones del proveedor. Para el gen subolesina, la transcriptasa reversa (RT)-PCR fue utilizada para amplificar el mARN extraído utilizando el kit EZ-10 Spin Column Total RNA Miniprep (Bio Basic.inc, Toronto, Canadá) de los diferentes aislados de $R$. microplus provenientes de Yucatán, Nayarit, Tamaulipas, Chiapas, Querétaro, Sinaloa y Veracruz, el cADN fue sintetizado con SuperScript III Firs-Strand Synthesis System Kit (Invitrogen, Carlsbad, CA, USA) de acuerdo a las intrucciones del proveedor.

Para la reacción de PCR se utilizó un volumen de reacción total $20 \mu \mathrm{L}$ : ADN $1 \mu \mathrm{L},(50$ ng/ $\mu \mathrm{L}$ ), $1 \mu \mathrm{L}$ de cada iniciador, $10 \mu \mathrm{L}$ de PCR Master Mix, Promega, Wisconsin, USA) y $7 \mu \mathrm{L}$ de agua libre de nucleasas.

La reacción se llevó a cabo bajo las siguientes condiciones: 1 ciclo de desnaturalización a $95^{\circ} \mathrm{C}$ por 3 minutos seguida de 34 ciclos de desnaturalización a $95^{\circ} \mathrm{C}$ por 30 segundos, alineamiento de $56^{\circ} \mathrm{C}$ por 30 segundos y una extensión a $72^{\circ} \mathrm{C}$ por 1 minuto y el paso final de extensión a $72^{\circ} \mathrm{C}$ por 5 minutos. 
Los productos de PCR se utilizaron para realizar la electroforesis en gel de agarosa a una concentración de 1.5\%. Los geles fueron observados en el fotodocumentador para verificar que las amplificaciones presentaran la medida correcta, $780 \mathrm{pb}$ para bmvdac y $442 \mathrm{pb}$ para subolesina. Las bandas de los amplicones esperados fueron cortados del gel de agarosa y purificados utilizando el kit comercial de purificación de ADN Wizard SV Gel and PCR CleanUp System (Promega, USA). El ADN purificado fue enviado a secuenciar por el método automatizado de Sanger al Instituto de Biotecnología (IBT) de la Universidad Nacional Autónoma de México (Cuernavaca, Morelos, México).

\section{Obtención de secuencias consenso, alineamiento múltiple y análisis bioinformático}

A las secuencias de nucleótidos obtenidas del análisis y ensamblaje del resultado de la secuenciación se les realizó un análisis de búsqueda de marco de lectura abierto u ORF (Open Reading Frame) mediante el algoritmo bioinformático ORF finder (http://www.ncbi.nlm.nih.gov/projects/gorf/) que busca en la secuencia de nucleótidos la presencia de codones de inicio y de paro necesarios para su traducción a aminoácidos. De esta forma se obtuvo la secuencia de aminoácidos predicha correspondiente a cada gen de los diferentes aislados. Los análisis siguientes fueron realizados con estas secuencias.

Las secuencias de los diferentes aislados seleccionados fueron analizadas mediante el programa de alineamiento multiple Clustal Omega y el programa BLAST (Basic Logical Aligment Search Tool) para la identificación de las regiones conservadas.

Para el análisis de identidad y similitud entre las diferentes secuencias se utilizó el programa SIAS (Secuence Identites and Similarities) http://imed.med.ucm.es/Tools/sias.

\section{Análisis filogenético}

Los árboles filogenéticos fueron costruídos basados en el Método UPGMA (Unweighted Pair Group Method using Arithmetic averages) utilizando el programa MEGAX64 (Molecular Evolutionary Genetics Analysis software) (Tamura et al., 2013). Para evaluar la confiabilidad de las topologías del árbol inferidas, los análisis se realizaron utilizando el método estadístico Bootstrap con 10000 réplicas (Felsenstein, 1985). Para bmvdac se utilizó como grupo externo a Ixodes scapularis con número de acceso de GenBank XP_002408065.1 y Amblyomma variegatum con número de acceso BK007268.1. Para subolesina se utilizó como grupo externo a 
Análisis de la variabilidad alélica de BmVDAC y Subolesina, dos candidatos vacunales contra Rhipicephalus microplus en aislados de México

Ixodes ricinus con número de acceso de GenBank ABA62325.1. y Amblyomma cajennense con número de acceso AGI44599.1.

\section{$\underline{\text { Resultados }}$}

Los aislados de $R$. microplus fueron obtenidos de diferentes zonas geográficas de México como se muestra en la Tabla 1.

Tabla 1. Localización de las zonas geográficas de México de aislados de garrapatas $R$. microplus incluidas en el presente estudio.

\begin{tabular}{|c|c|c|c|c|}
\hline \multirow{2}{*}{$\begin{array}{l}\text { Habitad de } \\
\text { las garrapatas }\end{array}$} & \multirow[t]{2}{*}{ Estado } & \multicolumn{2}{|c|}{ Genes amplificados } & \multirow{2}{*}{$\begin{array}{l}\text { Localización } \\
\text { (Latitud, longitud) }\end{array}$} \\
\hline & & $b m v d a c$ & subolesina & \\
\hline Noreste & Tamaulipas & $\checkmark$ & $\checkmark$ & $\begin{array}{l}22^{\circ} 39^{\prime} 35.6^{\prime \prime} \mathrm{N} \\
98^{\circ} 15^{\prime} 14.1^{\prime \prime} \mathrm{W}\end{array}$ \\
\hline Centro & Querétaro & $\checkmark$ & $\checkmark$ & $\begin{array}{l}21^{\circ} 11^{\prime} 00.03^{\prime \prime} \mathrm{N} \\
99^{\circ} 16^{\prime} 38.8^{\prime \prime} \mathrm{W}\end{array}$ \\
\hline Sureste & Tabasco & $\checkmark$ & & $\begin{array}{l}17^{\circ} 48^{\prime} 35.6^{\prime \prime} \mathrm{N} \\
93^{\circ} 23^{\prime} 42.8^{\prime \prime} \mathrm{W}\end{array}$ \\
\hline Noroeste & Sinaloa & $\checkmark$ & $\checkmark$ & $\begin{array}{c}23^{\circ} 17^{\prime} 18.8^{\prime \prime} \mathrm{N} \\
106^{\circ} 25^{\prime} 02.3^{\prime \prime} \mathrm{W}\end{array}$ \\
\hline Suroeste & Chiapas & $\checkmark$ & & $\begin{array}{l}16^{\circ} 18^{\prime} 11.8^{\prime \prime} \mathrm{N} \\
91^{\circ} 55^{\prime} 29.9^{\prime \prime} \mathrm{W}\end{array}$ \\
\hline Suroeste & Guerrero & $\checkmark$ & & $\begin{array}{c}18^{\circ} 22^{\prime} 01.0^{\prime \prime N} \\
100^{\circ} 37^{\prime} 57.6^{\prime \prime} \mathrm{W}\end{array}$ \\
\hline Oeste & Jalisco & $\checkmark$ & & $\begin{array}{r}19^{\circ} 56^{\prime} 33.4^{\prime \prime} \mathrm{N} \\
103^{\circ} 45^{\prime} 47.8^{\prime \prime} \mathrm{W}\end{array}$ \\
\hline Oeste & Nayarit & $\checkmark$ & $\checkmark$ & $\begin{array}{r}21^{\circ} 32^{\prime} 34.05^{\prime \prime} \mathrm{N} \\
104^{\circ} 56^{\prime} 71.09^{\prime \prime} \mathrm{V}\end{array}$ \\
\hline Sureste & Yucatán & & $\checkmark$ & $\begin{array}{l}20^{\circ} 52^{\prime} 56.82^{\prime \prime N} \\
89^{\circ} 37^{\prime} 55.85^{\prime \prime} \mathrm{W}\end{array}$ \\
\hline Este & Veracruz & & $\checkmark$ & $\begin{array}{l}20^{\circ} 05^{\prime} 12.5^{\prime \prime} \mathrm{N} \\
97^{\circ} 02^{\prime} 43.7^{\prime \prime} \mathrm{W}\end{array}$ \\
\hline
\end{tabular}


La secuencia utilizada como referencia para bmvdac corresponde a un fragmento de 819 pares de bases y va de los nucleótidos 1 al 819 de la cepa Media Joya de Tapalpa, Jalisco, reportada previamente (Rodríguez-Hernández et al., 2015), y de ese fragmento fueron amplificadas 750 pares de bases para el presente estudio. Este fragmento corresponde al $91.57 \%$ de la secuencia de referencia, y para subolesina la secuencia de referencia corresponde al fragmento de 442 pares de bases y va del nucleótido 1 al 442 de la cepa Munoz usada como referencia y publicada anteriormente (Almazán et al., 2010). De ese fragmento fueron amplificados 438 pares de bases que corresponden al $99.3 \%$ de la secuencia utilizada como referencia.

Para el alineamiento múltiple de bmvdac, se analizaron ocho aislados de $R$. microplus además de la secuencia de referencia reportada en GenBank (Fig. 1). En donde se observan las zonas conservadas entre las diferentes secuencias, los aislados que presentan diferencias en este alineamiento son, Jalisco, Tabasco, Sinaloa, Nayarit y Chiapas, las cuales presentan solamente un aminoácido diferente.

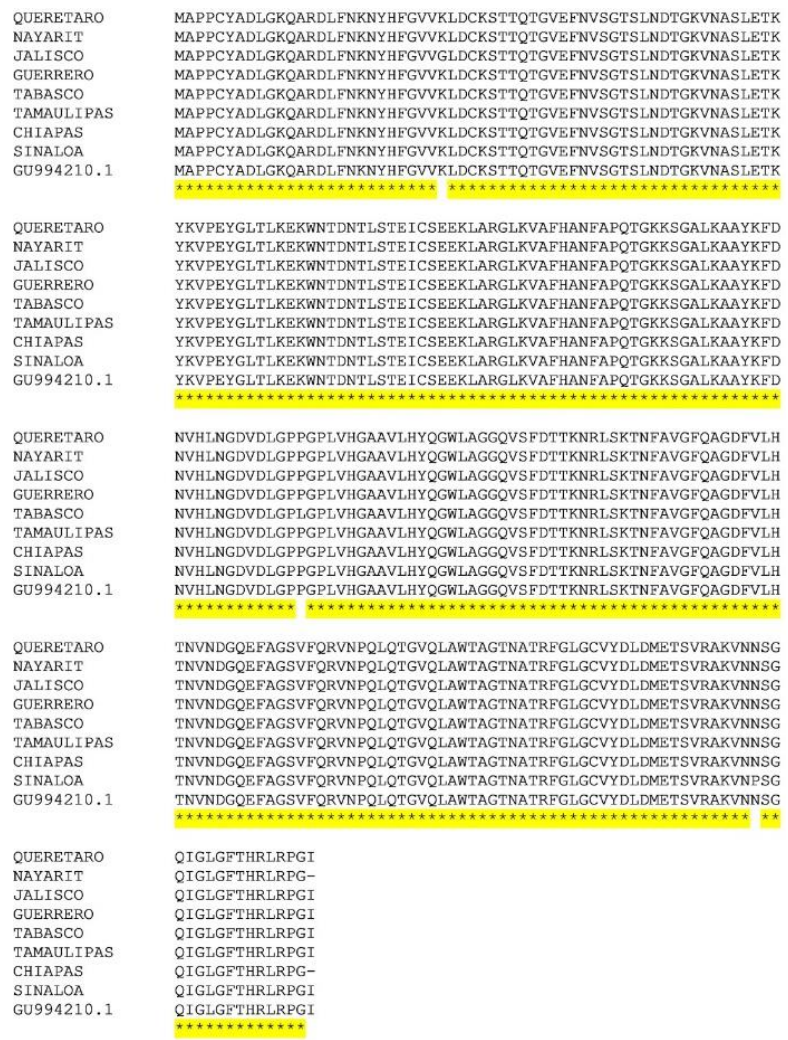

Fig. 1. Alineamiento múltiple de las secuencias de Jalisco, Tabasco, Chiapas, Nayarit, Querétaro, Tamaulipas, Guerrero, Sinaloa y la cepa de referencia GU994210.1. En amarillo están marcadas las regiones conservadas de las diferentes secuencias para el gen bmvdac. Se utilizó el programa CLUSTAL O (1.2.4) múltiple sequence alignment. 
En promedio de observa un porcentaje del $99.79 \%$ de identidad y similitud. En seis de las nueve secuencias utilizadas provenientes de las diferentes zonas geográficas de México, se observó un porcentaje tanto de identidad como de similitud del $99.87 \%$ en las secuencias de aminoácidos de Chiapas, Nayarit, Media Joya, Querétaro, Tamaulipas y Guerrero. En las secuencias provenientes de Jalisco, Tabasco y Sinaloa presentaron el $99.56 \%$ en identidad y similitud. En promedio se observa un porcentaje de variabilidad de $0.21 \%$ entre las secuencias de bmvdac, como se indica en Tablas 2 y 3.

Tabla 2. Porcentajes de identidad entre las secuencias de aminoácidos de los aislados provenientes de los diferentes estados de la República Mexicana para bmvdac. Se utilizó el programa SIAS, Sequences Identites and Similarities.

\begin{tabular}{|c|c|c|c|c|c|c|c|c|c|}
\hline AISLADOS & QUERÉTARO & NAYARIT & JALISCO & GUERRERO & TABASCO & TAMAULIPAS & CHIAPAS & SINALOA & GU994210.1. \\
\hline QUERÉTARO & $100 \%$ & & & & & & & & \\
\hline NAYARIT & $100 \%$ & $100 \%$ & & & & & & & \\
\hline JALISCO & $99.60 \%$ & $99.60 \%$ & $100 \%$ & & & & & & \\
\hline GUERRERO & $100 \%$ & $100 \%$ & $99.60 \%$ & $100 \%$ & & & & & \\
\hline TABASCO & $99.60 \%$ & $99.60 \%$ & $99.21 \%$ & $99.60 \%$ & $100 \%$ & & & & \\
\hline TAMAULIPAS & $100 \%$ & $100 \%$ & $99.60 \%$ & $100 \%$ & $99.60 \%$ & $100 \%$ & & & \\
\hline CHIAPAS & $100 \%$ & $100 \%$ & $99.60 \%$ & $100 \%$ & $99.60 \%$ & $100 \%$ & $100 \%$ & & \\
\hline SINALOA & $99.60 \%$ & $99.60 \%$ & $99.21 \%$ & $99.60 \%$ & $99.21 \%$ & $99.60 \%$ & $99.60 \%$ & $100 \%$ & \\
\hline GU994210.1. & $100 \%$ & $100 \%$ & $99.60 \%$ & $100 \%$ & $99.60 \%$ & $100 \%$ & $100 \%$ & $99.60 \%$ & $100 \%$ \\
\hline
\end{tabular}

Tabla 3. Porcentajes de Similitud entre las secuencias de aminoácidos de los aislados provenientes de los diferentes estados de la República Mexicana para bmvdac. Se utilizó el programa SIAS, Sequences Identites and Similarities.

\begin{tabular}{|c|c|c|c|c|c|c|c|c|c|}
\hline AISLADOS & QUERÉTARO & NAYARIT & JALISCO & GUERRERO & TABASCO & TAMAULIPAS & CHIAPAS & SINALOA & GU994210.1. \\
\hline QUERÉTARO & $100 \%$ & & & & & & & & \\
\hline NAYARIT & $100 \%$ & $100 \%$ & & & & & & & \\
\hline JALISCO & $99.60 \%$ & $99.60 \%$ & $100 \%$ & & & & & & \\
\hline GUERRERO & $100 \%$ & $100 \%$ & $99.60 \%$ & $100 \%$ & & & & & \\
\hline TABASCO & $99.60 \%$ & $99.60 \%$ & $99.21 \%$ & $99.60 \%$ & $100 \%$ & & & & \\
\hline TAMAULIPAS & $100 \%$ & $100 \%$ & $99.60 \%$ & $100 \%$ & $99.60 \%$ & $100 \%$ & & & \\
\hline CHIAPAS & $100 \%$ & $100 \%$ & $99.60 \%$ & $100 \%$ & $99.60 \%$ & $100 \%$ & $100 \%$ & & \\
\hline SINALOA & $99.60 \%$ & $99.60 \%$ & $99.21 \%$ & $99.60 \%$ & $99.21 \%$ & $99.60 \%$ & $99.60 \%$ & $100 \%$ & \\
\hline GU994210.1. & $100 \%$ & $100 \%$ & $99.60 \%$ & $100 \%$ & $99.60 \%$ & $100 \%$ & $100 \%$ & $99.60 \%$ & $100 \%$ \\
\hline
\end{tabular}

En el alineamiento múltiple de subolesina con siete diferentes aislados provenientes de Yucatán, Nayarit, Querétaro, Chiapas, Sinaloa, Veracruz y Tamaulipas y la cepa Muñoz de referencia (Fig. 2), se observan las zonas conservadas entre las diferentes secuencias, los aislados que presentan diferencias en este alineamiento son, Sinaloa, Nayarit y Yucatán. 


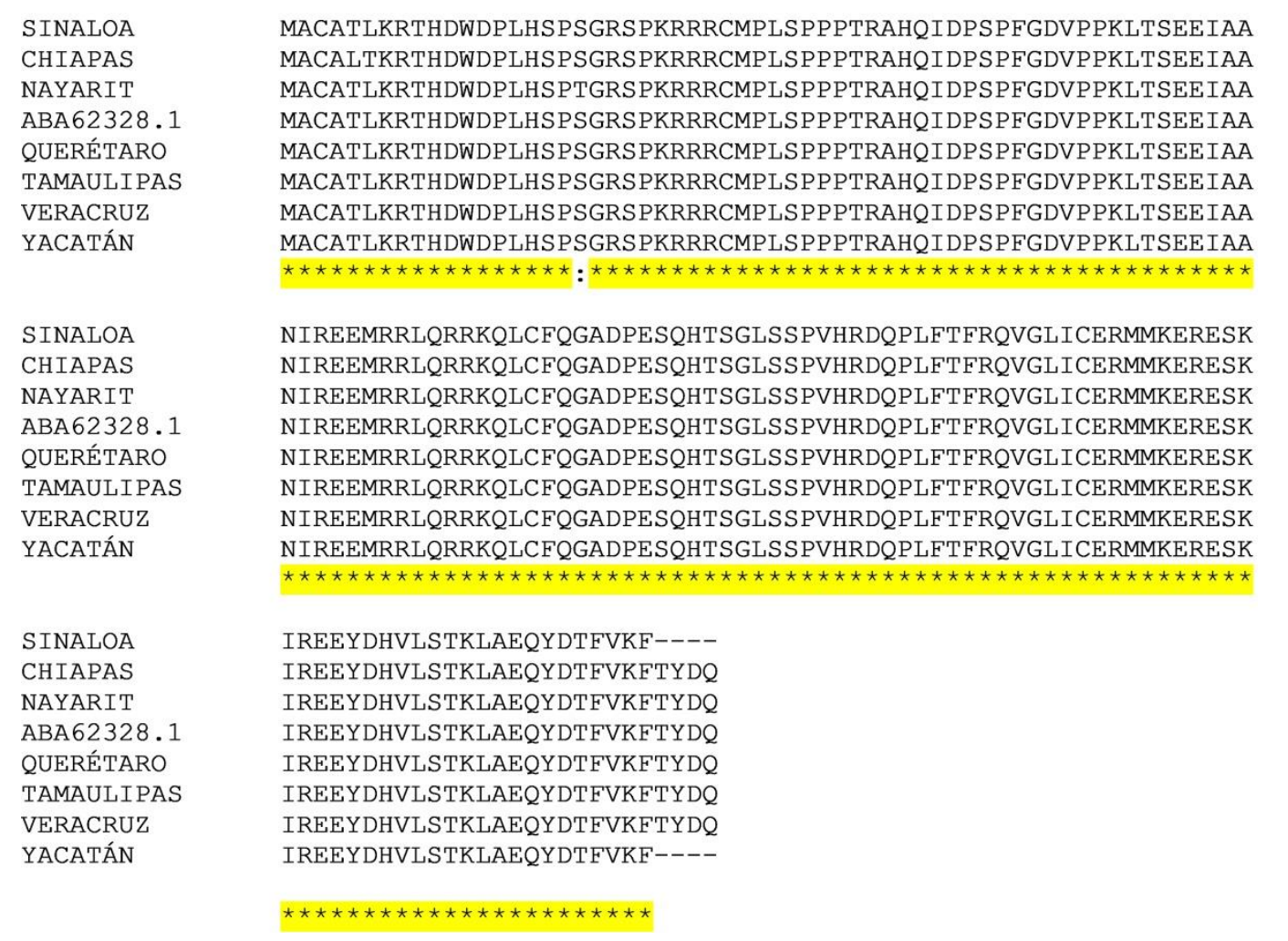

Fig. 2. Alineamiento multiple de las secuencias de Yucatán, Nayarit, Querétaro, Sinaloa, Veracruz, Tamaulipas y la cepa de referencia ABA62328.1. En amarillo se encuentran marcadas las regiones conservadas de las diferentes secuencias para el gen subolesina. Se utilizó el programa CLUSTAL O (1.2.4) múltiple sequence alignment.

Además, se observó un porcentaje promedio de identidad del $99.87 \%$ y similitud del $100 \%$ entre las secuencias de aminoácidos. De las ocho secuencias, siete de ellas presentaron procentaje de identidad del 99.91\%, mientras que para la secuencia de Nayarit el porcentaje fue de $99.39 \%$ (Tabla 4 y 5). La variabilidad promedio observada entre las secuencias de aminoácidos fue del $0.13 \%$.

\begin{tabular}{|c|c|c|c|c|c|c|c|c|}
\hline AISLADOS & ABA 62328.1 & CHIAPAS & NAYARIT & QUERÉTARO & TAMAULIPAS & VERACRUZ & SINALOA & YUCATÁN \\
\hline ABA62328.1 & $100 \%$ & & & & & & & \\
\hline CHIAPAS & $100 \%$ & $100 \%$ & & & & & & \\
\hline NAYARIT & $99.31 \%$ & $99.31 \%$ & $100 \%$ & & & & & \\
\hline QUERÉTARO & $100 \%$ & $100 \%$ & $99.31 \%$ & $100 \%$ & & & & \\
\hline TAMAULIPAS & $100 \%$ & $100 \%$ & $99.31 \%$ & $100 \%$ & $100 \%$ & & & \\
\hline VERACRUZ & $100 \%$ & $100 \%$ & $99.31 \%$ & $100 \%$ & $100 \%$ & $100 \%$ & & \\
\hline SINALOA & $100 \%$ & $100 \%$ & $99.30 \%$ & $100 \%$ & $100 \%$ & $100 \%$ & $100 \%$ & \\
\hline YUCATÁN & $100 \%$ & $100 \%$ & $99.30 \%$ & $100 \%$ & $100 \%$ & $100 \%$ & $100 \%$ & $100 \%$ \\
\hline
\end{tabular}

Tabla 4. Porcentajes de identidad entre las secuencias de aminoácidos de los aislados provenientes de los diferentes estados de la República Mexicana para subolesina. Se utilizó el programa SIAS, Sequences Identites and Similarities.

No 23, Vol. 11 (2), 2019. ISSN 2007 - 0705, pp.: 126 - 142 $-136-$ 


\begin{tabular}{|c|c|c|c|c|c|c|c|c|}
\hline AISLADOS & ABA62328.1 & CHIAPAS & NAYARIT & QUERÉTARO & TAMAULIPAS & VERACRUZ & SINALOA & YUCATÁN \\
\hline ABA62328.1 & $100 \%$ & & & & & & & \\
\hline CHIAPAS & $100 \%$ & $100 \%$ & & & & & & \\
\hline NAYARIT & $100 \%$ & $100 \%$ & $100 \%$ & & & & & \\
\hline QUERETARO & $100 \%$ & $100 \%$ & $100 \%$ & $100 \%$ & & & & \\
\hline TAMAULIPAS & $100 \%$ & $100 \%$ & $100 \%$ & $100 \%$ & $100 \%$ & & & \\
\hline VERACRUZ & $100 \%$ & $100 \%$ & $100 \%$ & $100 \%$ & $100 \%$ & $100 \%$ & & \\
\hline SINALOA & $100 \%$ & $100 \%$ & $100 \%$ & $100 \%$ & $100 \%$ & $100 \%$ & $100 \%$ & \\
\hline YUCATÁN & $100 \%$ & $100 \%$ & $100 \%$ & $100 \%$ & $100 \%$ & $100 \%$ & $100 \%$ & $100 \%$ \\
\hline
\end{tabular}

Tabla 5. Porcentajes de similitud entre las secuencias de aminoácidos de los aislados provenientes de los diferentes estados de la República Mexicana para subolesina. Se utilizó el programa SIAS, Sequences Identites and Similarities.

Tanto los alineamientos multiples como los porcentajes de identidad y similitud resultantes en el presente estudio muestran la presencia de zonas conservadas entre las diferentes secuencias tanto para BmVDAC como para Subolesina; con base en lo reportado por García et al. (1999) y Freeman et al. (2010), referente a que el polimorfismo puede ser la causa de la variabilidad en la eficacia de la vacuna con el antígeno Bm86. BmVDAC y Subolesina, por su parte, presentan potencial para ser considerados como candidatos vacunales para el control de la garrapata $R$. microplus en México.

Los análisis filogenéticos mostraron similitudes muy elevadas en el árbol diseñado utilizando el método UPGMA. En el filograma construido para $b m v d a c$ con los aislados incluidos en el presente estudio se observan tres ramificaciones principales (Fig. 3), en la primera de ellas se encuentran, Chiapas, la cepa de referencia GU994210.1, Tamaulipas, Guerrero, Nayarit y Querétaro, las cuales presentan una misma secuencia genética localizada en diferentes zonas geográficas. Por otra parte, Sinaloa comparte una gran similitud con Tabasco y Jalisco. La segunda y tercera ramificación están conformada por las secuencias utilizadas como grupo externo (Amblyomma variegatum e Ixodes Scapuraris). El porcentaje obtenido del método Bootstrap de 10000 réplicas es mayor al 50\% como se observa en los nodos respectivos (Xiong, 2006). 


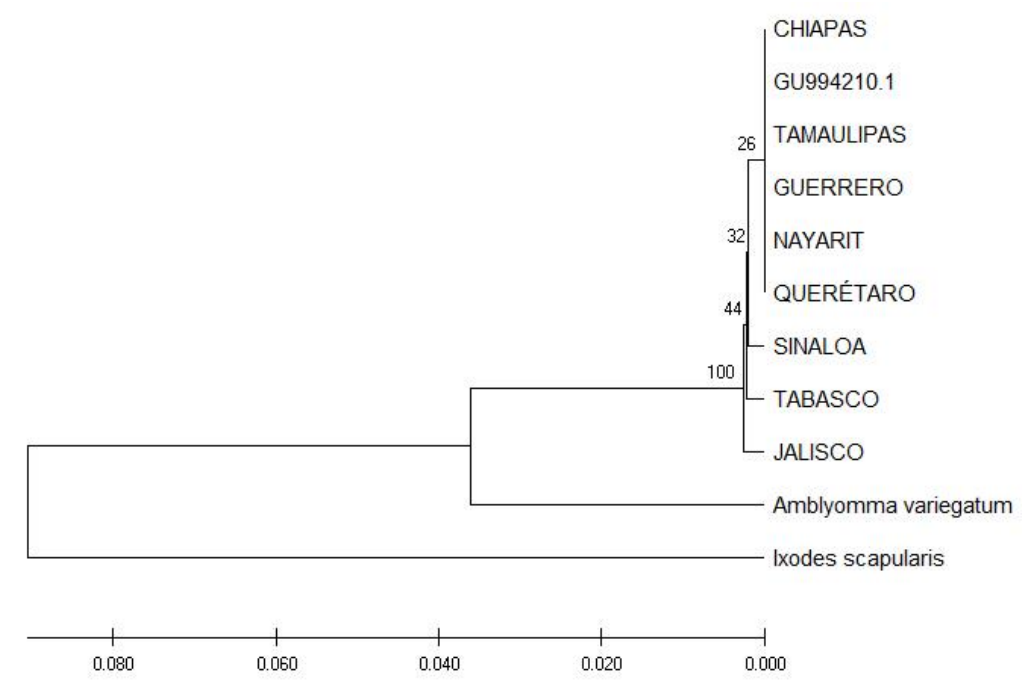

Fig. 3. Relación filogenética de los aislados de R. microplus para el gen bmvdac. El árbol filogenético fue construido utilizando el método UPGMA. Al lado izquierdo del nodo se encuentra el porcentaje de soporte del método Bootstrap utilizando 10000 réplicas.

El árbol filogenético para subolesina presenta tres ramificaciones (Fig. 4), en la primera, se encuentran una misma secuencia genética en los aislados de Sinaloa, Yucatán, Veracruz, Tamaulipas, Querétaro, Chiapas y la cepa de referencia ABA62328, por una parte y por la otra, se encuentra Nayarit. En la segunda y tercera ramificación se encuentra la secuencia de Amblyomma cajennense e Ixodes ricinus respectivamente, ambas utilizadas como grupo externo. El porcentaje obtenido del método Bootstrap de 10000 réplicas es del 82\%.

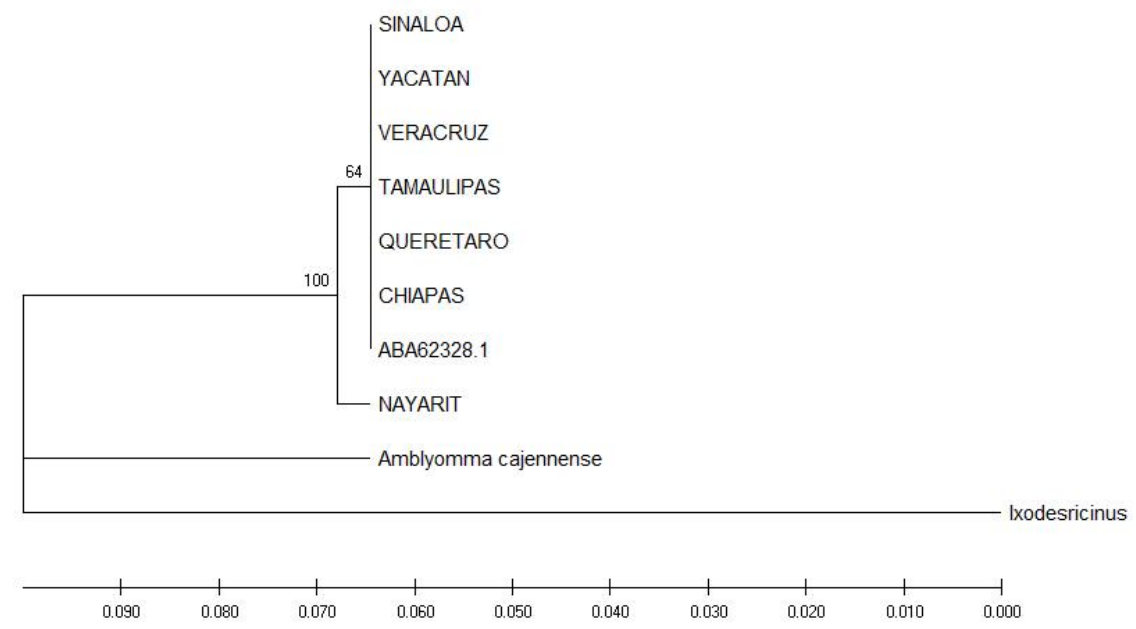

Fig. 1. Relación filogenética de los aislados de R. microplus para el gen subolesina. El árbol filogenético fue construido utilizando el método UPGMA. Al lado izquierdo del nodo se encuentra el porcentaje de soporte del método Bootstrap utilizando 10000 réplicas. 


\section{Discusión y Conclusiones}

Este reporte representa la primera investigación de la caracterización de la variabilidad alélica para para los genes bmvdac y subolesina, dos antígenos vacunales contra $R$. microplus entre aislados de diferentes estados en México. De acuerdo a lo reportado por Kaewmongkol et al., 2015, la variabilidad en la secuencia obtenida de la garrapata $R$. microplus para Bm86 es una posible explicación referente al rango de eficacia presentada en la vacuna contra este parásito, utilizando como antígeno la proteína recombinante Bm86. Debido a esto, para amplificar bmvdac y subolesina se seleccionaron aislados representativos de diferentes áreas geográficas de México, para de esta manera determinar la variabilidad entre las secuencias provenientes de diferentes estados del país.

De acuerdo a los datos publicados por García-García et al., 1999, la variabilidad superior al 3.4\% entre las diferentes secuencias es suficiente para producir una respuesta inmune ineficiente, por lo que es importante destacar que la variabilidad entre las secuencias de aminoácidos de los aislados en el presente estudio es de un $0.21 \%$ para bmvdac y para subolesina se observa una variabilidad del $0.13 \%$. Por consiguiente, con este análisis se considera que bmvdac y subolesina son antígenos que se encuentran conservados en las secuencias de las poblaciones de $R$. microplus de las diferentes zonas geográficas del país.

En el árbol filogenético para bmvdac se observa que las secuencias incluidas en la construcción del árbol presentan una misma secuencia genética localizada en seis diferentes zonas geográficas, lo cual indica que su alto porcentaje de similitud se encuentra disperso en las diferentes poblaciones de R. microplus; se observan diferencias en las secuencias de Jalisco, localizado en la zona oeste de México, Tabasco en el sureste y Sinaloa en el noroeste. En el árbol filogenético para subolesina, de las ocho secuencias incluidas para la construcción del árbol, siete de ellas provenientes de diferentes zonas geográficas comparten una misma secuencia genética, mientras que solo una de ellas, en este caso Nayarit localizado en la zona oeste del país, presenta variabilidad en su secuencia. Las diferencias observadas tanto bmvdac como en subolesina pueden ser debidas a la movilización del ganado del sur del país hacia los estados del norte y no a las diferentes zonas geográficas de donde provienen los aislados utilizados en el presente estudio, lo cual difiere de lo sugerido por De la Fuente et al., 2007. El porcentaje obtenido del método Bootstrap utilizando 10000 réplicas tanto para bmvdac como para subolesina es superior al 50\%, lo cual indica un soporte estadístico fiable, de acuerdo a lo reportado por Xion et al., 2006. 
Considerando la baja variabilidad alélica presentada entre las secuencias de los aislados

de $R$. microplus de los diferentes estados de México, se concluye que tanto BmVDAC como Subolesina son antígenos que presentan secuencias altamente conservadas en aislados de México, por lo que pueden ser considerados como potenciales candidatos vacunales de acuerdo a lo reportado por Freeman et al., 2010, donde sugiere que existe una correlación inversa entre la eficacia de la vacuna y la variación del locus; por lo tanto, esta información es relevante para la selección de antígenos empleados en las vacunas contra garrapatas $R$. microplus. Se sugiere la realización de evaluaciones in vivo para determinar su efectividad como inmunógenos.

\section{Agradecimientos}

A CONACyT y al Fondo de proyectos especiales de rectoría (FOPER) por el apoyo para la realización de este proyecto.

\section{$\underline{\text { Referencias }}$}

Abbas, Rao Z., Muhammad Arfan Zaman, Douglas D. Colwell, John Gilleard, y Zafar Iqbal. (2014). Acaricide resistance in cattle ticks and approaches to its management: The state of play. Veterinary Parasitology 203 (1-2): 6-20.

Almazán, Consuelo, Rodolfo Lagunes, Margarita Villar, Mario Canales, Rodrigo Rosario-Cruz, Frans Jongejan, y José de la Fuente. (2010). Identification and Characterization of Rhipicephalus (Boophilus) Microplus Candidate Protective Antigens for the Control of Cattle Tick Infestations. Parasitology Research 106 (2): 471-79.

Antunes, Sandra, Octavio Merino, Juan Mosqueda, Juan A. Moreno-Cid, Lesley Bell-Sakyi, Rennos Fragkoudis, Sabine Weisheit, et al. (2014). Tick capillary feeding for the study of proteins involved in tick-pathogen interactions as potential antigens for the control of tick infestation and pathogen infection. Parasites \& Vectors 7: 42.

De La Fuente, J., y K. M. Kocan. (2006). Strategies for Development of Vaccines for Control of Ixodid Tick Species. Parasite Immunology 28 (7): 275-83.

Felsenstein, Joseph. (1985). Phylogenies and the Comparative Method. The American Naturalist 125 (1): 1-15. 
Freeman, Jeanne M., Ronald B. Davey, Lowell S. Kappmeyer, Diane M. Kammlah, y Pia U. Olafson. (2010). Bm86 midgut protein sequence variation in South Texas cattle fever ticks. Parasites \& Vectors 3: 101.

Fuente, José de la, Consuelo Almazán, Mario Canales, José Manuel Pérez de la Lastra, Katherine M. Kocan, y Peter Willadsen. (2007). A Ten-Year Review of Commercial Vaccine Performance for Control of Tick Infestations on Cattle. Animal Health Research Reviews 8 (1): $23-28$.

Fuente, José de la, y Katherine M. Kocan. (2003). Advances in the Identification and Characterization of Protective Antigens for Recombinant Vaccines against Tick Infestations. Expert Review of Vaccines 2 (4): 583-93.

Fuente, José de la, Christine Maritz-Olivier, Victoria Naranjo, Patricia Ayoubi, Ard M. Nijhof, Consuelo Almazán, Mario Canales, et al. (2008). Evidence of the Role of Tick Subolesin in Gene Expression. BMC Genomics 9: 372.

García-García, J. C., I. L. Gonzalez, D. M. González, M. Valdés, L. Méndez, J. Lamberti, B. D’Agostino, et al. (1999). Sequence Variations in the Boophilus Microplus Bm86 Locus and Implications for Immunoprotection in Cattle Vaccinated with This Antigen. Experimental \& Applied Acarology 23 (11): 883-95.

García-García, J. C., A. Soto, F. Nigro, M. Mazza, M. Joglar, M. Hechevarría, J. Lamberti, y J. de la Fuente. (1998). Adjuvant and Immunostimulating Properties of the Recombinant Bm86 Protein Expressed in Pichia Pastoris. Vaccine 16 (9-10): 1053-55.

Graf, J. F., R. Gogolewski, N. Leach-Bing, G. A. Sabatini, M. B. Molento, E. L. Bordin, y G. J. Arantes. (2004). Tick Control: An Industry Point of View. Parasitology 129 Suppl: S427442.

Jonsson, N. N. (2006). The Productivity Effects of Cattle Tick (Boophilus Microplus) Infestation on Cattle, with Particular Reference to Bos Indicus Cattle and Their Crosses. Veterinary Parasitology 137 (1-2): 1-10.

Kaewmongkol, S., G. Kaewmongkol, N. Inthong, N. Lakkitjaroen, T. Sirinarumitr, C. M. Berry, N. N. Jonsson, R. W. Stich, y S. Jittapalapong. (2015). Variation among Bm86 Sequences in Rhipicephalus (Boophilus) Microplus Ticks Collected from Cattle across Thailand. Experimental \& Applied Acarology 66 (2): 247-56. 
Manjunathachar, Haranahalli Vasanthachar, Buddhi Chandrasekaran Saravanan, Manickam Kesavan, Kumaragurubaran Karthik, Prakashkumar Rathod, Marappan Gopi, Paramasivam Tamilmahan, y Bharemara Lingaraju Balaraju. (2014). Economic importance of ticks and their effective control strategies. Asian Pacific Journal of Tropical Disease 4 (Supplement 2): S770-79.

Prudencio, Carlos R., Andrea O. M. Marra, Rone Cardoso, y Luiz R. Goulart. (2010). Recombinant Peptides as New Immunogens for the Control of the Bovine Tick, Rhipicephalus (Boophilus) Microplus». Veterinary Parasitology 172 (1-2): 122-31.

Rodríguez-Hernández, Elba, Juan Mosqueda, Gloria León-Ávila, Elizabeth J. Castañeda-Ortiz, María Elizbeth Álvarez-Sánchez, Alejandro D. Camacho, Alberto Ramos, y Minerva Camacho-Nuez. (2015). BmVDAC Upregulation in the Midgut of Rhipicephalus Microplus, during Infection with Babesia Bigemina. Veterinary Parasitology 212 (3-4): 368-74.

Sonenshine, Daniel E., y R. Michael Roe. (2013). Biology of Ticks. OUP USA.

Sossai, Sidimar, Ana P. Peconick, Policarpo A. Sales-Junior, Francismar C. Marcelino, Marlene I. Vargas, Elisangela S. Neves, y Joaquín H. Patarroyo. (2005). Polymorphism of the Bm86 Gene in South American Strains of the Cattle Tick Boophilus Microplus. Experimental \& Applied Acarology 37 (3-4): 199-214.

Taheri, M., S. Nabian, M. Ranjbar, R. Mazaheri Nezhad, A. Gerami Sadeghian, y A. Sazmand. (2014). Study of Vitellogenin in Boophilus Annulatus Tick Larvae and Its Immunological Aspects. Tropical Biomedicine 31 (3): 398-405.

Tamura, Koichiro, Glen Stecher, Daniel Peterson, Alan Filipski, y Sudhir Kumar. (2013). MEGA6: Molecular Evolutionary Genetics Analysis Version 6.0. Molecular Biology and Evolution 30 (12): 2725-29.

Walker, Alan R. (2009). Bowman A, Nuttall P: Ticks: Biology, Disease and Control. Parasites \& Vectors 2: 1.

Xiong, Jin. (2006). Essential Bioinformatics. Cambridge University Press. 